\title{
Editorial
}

\section{Intestinal Perforations in Very Low Birth Weight Infants: Timing May Be More Important Than You Think}

\author{
Phillip V. Gordon, MD, PhD \\ Joshua T. Attridge, MD
}

Journal of Perinatology (2005) 25, 561-562. doi:10.1038/sj.jp.7211372

In this edition of The Journal of Perinatology, the manuscript by Adesanya et al. ${ }^{1}$ provides the first published neurodevelopmental outcomes in very low birth weight infants who acquire intestinal perforation (IP). At first glance, the data are very concerning for IP acquired secondary to necrotizing enterocolitis (NEC) but less so for spontaneous intestinal perforation (SIP). This difference is likely to be real. However, as we will discuss shortly, we think this difference may be less robust than the manuscript suggests. Despite this, the authors are to be congratulated for having broken new ground, particularly so, for having been the first to report neurodevelopmental outcomes in infants with SIP.

There is good reason to ask if there might be a relationship between a hole in the bowel and untoward neurodevelopment. One must wonder what the effect of succus-induced peritonitis is upon the premature brain, particularly when the remedy for the source of systemic inflammation is not definitive (as with abdominal drainage). The answer may be forthcoming. Dr. Martin Blakely's prospective NICHD study is about to complete data collection at 18 to 22 months follow-up and preliminary reports indicate that infants with IP and initial abdominal drainage have worse neurodevelopmental outcomes than when compared to those treated with immediate laparotomy. ${ }^{2}$

More recently, another NICHD study has demonstrated that infants with surgical NEC have significantly worse neurodevelopmental outcomes than infants treated for medical NEC. ${ }^{3}$ When coupled with Adesanya et al.'s ${ }^{1}$ findings, it fast becomes clear that IP secondary to NEC carries a grave prognosis for neurodevelopment. However, there are no previous data available that examine outcomes for IP secondary to SIP — a fact that makes Adesanya's et al.'s ${ }^{1}$ manuscript of special interest.

Perhaps the first distinction to make is the evidence that SIP and NEC are two different diseases. In 1999, Gordon et al. ${ }^{4}$ first

Department of Pediatrics, UVA Health System, Charlottesville, VA, USA.

Address correspondence and reprint requests to Phillip V. Gordon, MD, PhD, Department of Pediatrics, University of Virginia Health Science Center, Division of Neonatology, PO Box 800386, Charlottesville, VA 22908, USA. demonstrated that SIP has unique histopathology. Similar to congenital segmental absence of the muscularis externa, SIP has focal necrosis of the muscularis but also has concomitant mucosal hypertrophy. Similar findings have since been independently confirmed by three other groups on as many continents. ${ }^{5-7}$ The histopathology of SIP looks nothing like NEC and speculation that it is early NEC or somehow is on the spectrum of NEC has no biological basis that we are aware of. Unfortunately, this does not mean that SIP and NEC can always be distinguished on clinical presentation alone. In Blakely's ${ }^{2}$ prospective study, physicians were correct in diagnosing SIP 83\% of the time (based on surgical confirmation). This task is much more challenging in a retrospective study.

We think that one of the most useful data elements for retrospectively discerning between NEC and SIP is the day of diagnosis (although Adesanya et al. ${ }^{1}$ concludes that this is not the case). While surgical exploration and histopathology is the gold standard today, during 1996 to 1999 few pathologists were aware of the diagnosis of SIP and we suspect there was a tendency throughout the country for a high NEC false-positive rate. Likewise, as the prevalence of SIP was rapidly increasing during this period, clinicians were also struggling with the diagnoses of SIP versus NEC. Since aggressive early feeding was reported by the authors to be the norm at Brenner Children's Hospital during this study period, perforated infants could theoretically have also acquired pneumatosis and NEC as a secondary complication if SIP went undiagnosed. Thus confusion and overlap between the diseases was likely during this epoch.

We have recently derived an 8-year database of infants $\leq 1000 \mathrm{~g}$ from the University of Virginia Children's Hospital from 1996 to 2004 and used it to examine the day of diagnosis for surgical NEC and SIP (Figure 1). In contrast to Adesanya et al., ${ }^{1}$ we find that this data element is remarkably accurate in discerning SIP from surgical NEC in aggregate. When comparing the two scatterplots, one can see that the cohort comparisons differ, not in the early clustering of SIP, but in Adesanya's skewed distribution of NEC towards early diagnosis. We have been collaborating with Reese Clark on the Pediatrix database (where the number of SIP cases from 1998 to 2004 outnumbers the total number of published cases in the literature) and the same bimodal pattern of IP timing was also evident in that national data set (data not shown, manuscript in submission).

Our data sets confirm that the timing of IP diagnosis is associated with its etiology. Thus, it seems likely that the NEC IP 


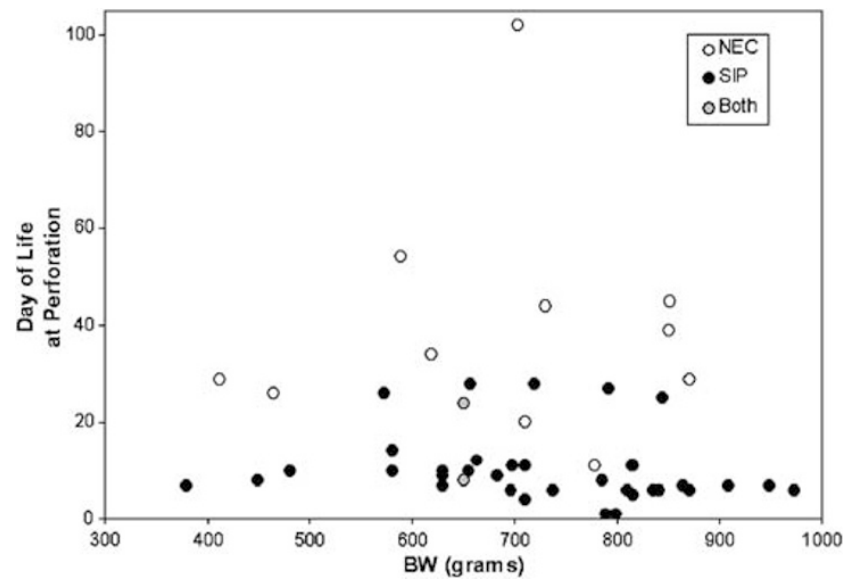

Figure 1. Scatterplot of the day of diagnosis versus birth weight in infants with spontaneous intestinal perforation (SIP — black circles) and surgical necrotizing enterocolitis (NEC — white circles). This is an 8-year cohort of $\leq 1000 \mathrm{~g}$ birth weight patients from the University of Virginia Children's Hospital derived from 1996 to 2004. One patient acquired both diseases (gray circles). In this cohort, SIP occurred earlier in life than NEC and was more that twice as prevalent. SIP predominated and NEC was rare in the first 2 weeks of life, whereas NEC predominated and SIP was less common after 2 weeks of life. We note that $66 \%$ of all SIP cases presenting after 2 weeks of life were occult in their manifestations (i.e., they never had pneumoperitoneum on Xray) and were typically diagnosed following a prolonged period of clinical suspicion.

cohort in the Adesanya paper is contaminated with SIP cases. What might this mean for the conclusions of the paper? First, the SIP cohort should be relatively homogenous based on their scatterplot and so one clear conclusion is that some SIP patients can have relatively optimistic neurodevelopmental outcomes at 1 year of age. Second, some SIP patients are likely contaminating the NEC cohort and might be contributing to the decreased MDI and PDI values or falsely elevating them. It seems more likely that SIP would be contributing to depressed scores and that these infants were sicker and therefore misdiagnosed or confounded by additional diagnoses (anecdotally, SIP was often diagnostically cherry-picked as those infants with blue bellies who did not present with signs of clinical instability in the 1990s, versus sicker infants presenting in the same window of time whose findings were felt to be more consistent with sepsis or NEC).

In our University of Virginia database, we looked at periventricular leukomalacia (PVL) on discharge ultrasounds and found that there was a significant increase in PVL within SIP infants compared with age-matched controls when using logisticregression analysis to exclude other risk factors (manuscript in submission). The magnitude of our findings (an odds ratio $>4$ for $\mathrm{PVL}$ ), suggest that a greater degree of neurodevelopmental perturbation might have been expected in Adesanya et al.' ${ }^{1}$ SIP cohort.

Adesanya et al. ${ }^{1}$ have not oversold their results. They do a good job of describing the limitations of their data, including the problems inherent with retrospective assignment of diagnosis. However, we think that day of diagnosis is a more important data element than they concluded and that it is a reliable criterion for assessing the diagnostic validity of IP data sets.

\section{References}

1. Adesanya OA, O'Shea TM, Turner CS, et al. Intestinal peforation in very low birth weight infants: growth and neurodevelopment at one year of age. J Perinatol 2005;25:583-89.

2. Blakely ML, Lally KP, McDonald S, et al. NEC Subcommittee of the NICHD Neonatal Research Network Postoperative outcomes of extremely low birthweight infants with necrotizing enterocolitis or isolated intestinal perforation: a prospective cohort study by the NICHD Neonatal Research Network. Ann Surg 2005;241(6):984-9; discussion 989-94.

3. Hintz SR, Kendrick DE, Stoll BJ, , et al., NICHD Neonatal Research Network. Neurodevelopmental and growth outcomes of extremely low birth weight infants after necrotizing enterocolitis. Pediatrics 2005;115(3):696-703.

4. Gordon PV, Rutledge J, Sawin R, Thomas S, Woodrum D. Early postnatal dexamethasone increases the risk of focal small bowel perforation in extremely low birth weight infants. J Perinatol 1999;19(8 Part 1):573-7.

5. Tatekawa Y, Muraji T, Imai Y, Nishijima E, Tsugawa C. The mechanism of focal intestinal perforations in neonates with low birth weight. Pediatr Surg Int 1999;15(8):549-52.

6. Hwang H, Murphy JJ, Gow KW, Magee JF, Bekhit E, Jamieson D. Are localized intestinal perforations distinct from necrotizing enterocolitis? J Pediatr Surg 2003;38(5):763-7.

7. Holland AJ, Shun A, Martin HC, Cooke-Yarborough C, Holland J. Small bowel perforation in the premature neonate: congenital or acquired? Pediatr Surg Int 2003;19(6):489-94. 